\section{P16.23 CLINICAL AND EPIDEMIOLOGICAL CHARACTERISTICS ASSOCIATED WITH UNFAVOURABLE TUBERCULOSIS TREATMENT OUTCOMES IN TB-HIV CO-INFECTED PATIENTS IN BRAZIL: A HIERARCHICAL POLYTOMOUS ANALYSIS}

${ }^{1}$ TN Prado*, ${ }^{2} J V$ Rajan, ${ }^{1}$ AE Miranda, ${ }^{1} E S$ Dias, ${ }^{1}$ LB Cosme, ${ }^{3}$ LG Possuelo, ${ }^{4} \mathrm{MN}$ Sanchez, ${ }^{1}$ EL Maciel. ${ }^{1}$ Federal University of Espirito Santo; ${ }^{2}$ University of California-San Francisco; ${ }^{3}$ University of Santa Cruz Do Sul; ${ }^{4}$ University of Brasilia

\subsection{6/sextrans-2015-052270.570}

Introduction TB-HIV co-infected patients have worse treatment outcomes than non-coinfected patients. How clinical characteristics of $\mathrm{TB}$ and patient socioeconomic characteristics influence these outcomes is poorly understood. We identified clinical and epidemiological characteristics associated with unfavourable treatment outcomes in TB-HIV co-infected patients in Brazil.

Methods TB-HIV cases reported in the Brazilian information system (SINAN) between January 1, 2001 and December 31, 2011 were identified and categorised by TB treatment outcome (cure, default, death and development of MDR TB). We modelled treatment outcome as a function of clinical characteristics of TB and patient socioeconomic characteristics using polytomous regression. For each possible outcome (default, death from $\mathrm{TB}$, death from other case, death from MDR-TB) the reference outcome was cure.

Results Patients aged 15-19 (OR = 2.86; 95\% CI: 2.09-3.91) and $20-39$ years old $(\mathrm{OR}=2.30 ; 95 \% \mathrm{CI}: 1.81-2.92)$ were more likely to default on TB treatment than those aged $0-14$ years old. In contrast, patients aged $\geq 60$ years were more likely to die from $\mathrm{TB}(\mathrm{OR}=2.22 ; 95 \% \mathrm{CI}: 1.43-3.44)$ or other causes $(\mathrm{OR}=2.86$; 95\% CI: 2.14-3.83). Black patients were more likely to default on $\mathrm{TB}$ treatment $(\mathrm{OR}=1.33$; 95\% CI: 1.22-1.44) and die from TB (OR $=1.50 ; 95 \% \mathrm{CI}: 1.29-1.74)$. Patients with $\geq 8$ years of education were less likely to default on $\mathrm{TB}$ treatment $(\mathrm{OR}=0.68 ; 95 \% \mathrm{CI}: 0.63-0.74)$, die from $\mathrm{TB}$ $(\mathrm{OR}=0.82 ; 95 \% \mathrm{CI}: 0.71-0.94)$ and die from other causes $(\mathrm{OR}=0.78 ; 95 \% \mathrm{CI}: 0.71-0.84)$. Finally, alcoholism was associated with all unfavourable outcomes: default $(\mathrm{OR}=1.94$; 95\% CI: $1.73-2.17)$, death due to $\mathrm{TB}(\mathrm{OR}=1.46 ; 95 \% \mathrm{CI}$ : $1.25-1.71)$, death due to other causes $(\mathrm{OR}=1.38 ; 95 \% \mathrm{CI}$ : 1.21-1.57) and MDR-TB (OR $=2.29$; 95\% CI: 1.46-3.58).

Conclusion Socio-economic vulnerability has a significant effect on treatment outcomes among TB-HIV co-infected patients in Brazil. Enhancing social support and incorporation of alcohol abuse screening and counselling into current TB surveillance programs and targeting interventions to specific age groups are specific interventions that could improve treatment outcomes. Disclosure of interest statement Nothing to declare.

\section{P16.24 A 20-YEAR RETROSPECTIVE COHORT STUDY OF TB INFECTION AMONG THE HILL TRIBE HIVIAIDS POPULATIONS, THAILAND}

Tawatchai Apidechkul* ${ }^{*}$ School of Health Science, Mae Fah Luang University, Chiang Rai

\subsection{6/sextrans-2015-052270.571}

Background A retrospective cohort study was conducted to determine the situation, trend, and possible risk factors of TB infection, and factors related to the health status among the HIV/AIDS hill tribe in northern Thailand.

Methods A systematic data-reviewing approach was used to identify the information from the rosters of ARV clinics, OPD cards, and laboratory reports from 16 hospitals in Chiang Rai Province, Thailand. The data were collected from the first reported HIV/AIDS case of the hill tribe to the end of 2010. A chi-square test and logistic regression models were used to identify associations at the significance level of alpha $=0.05$.

Results A total of 3,130 cases were included in the study. The majority of patients were Akha (46.0\%) followed by Lahu (19.7\%), $54.6 \%$ were males, $44.6 \%$ were $26-35$ years old, and $25.2 \%$ were $36-45$ years old. The peak period of HIV/AIDS infection among the hill tribes was from 2001-2005, during which occurred in $43.9 \%$ of all cases, followed by $33.7 \%$ from 2006-2010. The recorded occupations were $44.8 \%$ agricultural and $32.2 \%$ traders. The major risk factor of HIV infection was sexual intercourse $(91.7 \%) ; 33.3 \%$ were still alive at the date of data collection, $30.7 \%$ were diagnosed with pulmonary $\mathrm{TB}$, $76.0 \%$ did not receive ARV and 9.1\% had been checked for CD4 level. The Lisu hill tribe HIV/AIDS individuals had a greater risk of $\mathrm{TB}$ infection than did Lahu individuals $\left(\mathrm{OR}_{\mathrm{adj}}=\right.$ $1.50,95 \% \mathrm{CI}=1.04-2.16)$. Females had a greater risk of $\mathrm{TB}$ infection than did males $\left(\mathrm{OR}_{\mathrm{adj}}=1.22,95 \% \mathrm{CI}=1.01-1.49\right)$; being classified as symptomatic HIV group was a protective factor of $\mathrm{TB}$ with $\mathrm{OR}_{\mathrm{adj}}=0.18(95 \% \mathrm{CI}=0.11-0.29)$; and not having received the ARV was also a protective factor with $\mathrm{OR}_{\text {adj }}$ $=0.06(95 \% \mathrm{CI}=0.05-0.08)$. The patients who had been diagnosed with HIV infection during 2001-2005 and 2006-2010 had a greater risk of TB infection than did those who were diagnosed from 1990-1995, with $\mathrm{OR}_{\mathrm{adj}}=21.39$ (6.59-69.42) and 13.70 (4.19-44.73), respectively.

Conclusions Thailand needs to create a TB and HIV/AIDS surveillance system for hill tribe populations to determine the situation and trend and to develop an appropriate model for providing care at the earlier stage of HIV/AIDS infection to prevent later TB infection.

\section{P16.25 THE INCIDENCE AND ASSOCIATED FACTORS OF HERPES ZOSTER AMONG HIV INFECTED PATIENTS: A POPULATION-BASED RETROSPECTIVE COHORT STUDY IN TAIWAN, 2000 THROUGH 2010}

${ }^{1} \mathrm{~K}$ Han Chang ${ }^{*},{ }^{2} \mathrm{C}$ Yen Chin, ${ }^{3} \mathrm{~K}$ Nai Ying. ${ }^{1}$ ChiMei Medical Center, Tainan, Taiwan; ${ }^{2}$ The Department of Nursing, Institute of Allied Health Sciences, College of Medicine, National Cheng Kung University and Hospital, Tainan, Taiwan; ${ }^{3}$ Department of Nursing, Public Health, and Allied Health, National Cheng-Kung University, Tainan

\subsection{6/sextrans-2015-052270.572}

Introduction Highly active antiretroviral therapy (HAART) has been demonstrated to be effective in decreasing the incidence of opportunistic infections, AIDS, and death among patients with HIV infection. Herpes zoster (HZ) infection is common among patients with HIV infection. However, the impact of HAART on the incidence of $\mathrm{HZ}$ infection is not well understood.

Methods This nationwide, population-based, retrospective cohort study was conducted using Taiwan National Health Insurance Research Database (NHIRD) from 2000-2010. The NHIRD identified 15,112 patients with HIV infection. The incidence rates were standardised according to age based on the 2000 WHO standard population. Cox proportional hazards models were used to assess the effect of HAART on the incidence of $\mathrm{HZ}$ infection among patients with HIV infection.

Results The average incidence of the first episode of $\mathrm{HZ}$ after the diagnosis of HIV infection was estimated at 5.07 people/100 person-years. Multivariate Cox proportional hazards model showed that history of $\mathrm{HZ}$ infection (adjusted hazard ratio 
[aHR]: 6.76; 95\% CI, 5.19-8.82) and administration of HAART (aHR: 1.53 ; 95\% CI, 1.28-1.84) were the factors associated with HZ. Life-table method was used to divide the duration of HIV from diagnosis into two phases, namely, $\leq 4$ years and $>4$ years. Initiation of HAART within 4 years of HIV diagnosis was associated with an increased risk of $\mathrm{HZ}$ (HR: 1.79, 95\% CI: $1.48-2.16, \mathrm{p}<0.0001)$ and after 4 years of HIV diagnosis was associated with a decreased risk of $\mathrm{HZ}$ infection (HR: 0.60, 95\% CI: 0.47-0.78, p < 0.0001). Among HIV-infected patients on HAART, with $\geq 85 \%$ adherence was showed significantly lower risk of developing HZ (HR: 0.40, 95\% CI: 0.19-0.85, $\mathrm{p}<0.001)$.

Conclusion With high level of HAART adherence, it had significantly lowered HZ infection risk. Therefore, we suggested emphasising the importance of early treatment and HAART adherence.

\section{P16.26 HUMAN IMMUNODEFICIENCY VIRUS (HIV) - MYCOBACTERIUM TUBERCULOSIS (TB) CO-INFECTION IN SRI LANKA}

Dpcka Lal*. National Hospital of Sri Lanka

\subsection{6/sextrans-2015-052270.573}

Background TB and HIV co-infection is considered to occur worldwide. Immunosuppression by HIV makes patients vulnerable to be infected with $\mathrm{TB}$ and they are more prone to get severe disease. Prevalence of TB and HIV in Sri Lanka is $4.2 \%$ and $<0.1 \%$ respectively. Relationship between HIV and TB is not yet clearly defined in Sri Lanka. The objective of this preliminary study is to describe the epidemiology of HIV - TB coinfection in Sri Lanka.

Methods 54 sexually active patients with histopathologically or microbiologically proven Tuberculosis were screened for HIV with ELISA antibody test. Positive ELISA was confirmed by western blot test.

Results Patients were 17 to 54 years of age. Male: Female = 33:21. 38 and 16 patients had pulmonary and extra pulmonary TB respectively. Only 02 male patients had positive ELISA test for HIV but both were negative for western blot test.

Conclusion HIV - TB co-infection is not a significant occurrence in Sri Lanka yet. There for HIV should not be considered as an important predisposing factor for TB in Sri Lanka and It is not rational to screen all TB patients for HIV as it is not cost effective for a resource poor country.

Disclosure of interest Nothing to disclose.

\section{P16.27 FRAMINGHAM CHD AND CVDS RISK EQUATIONS IN HIV AND HIV/HCV POPULATION: A COMPARISON STUDY AMONG MALAYSIAN HIV INFECTED SUBJECTS ON ART}

${ }^{1} \mathrm{~N}$ Hejazi, ${ }^{1} \mathrm{R}$ Rajikan, ${ }^{2} \mathrm{CLK}$ Choong. ${ }^{1}$ National University of Malaysia; ${ }^{2}$ Sungai Buloh Hospital

\subsection{6/sextrans-2015-052270.574}

Introduction The possible underestimated risks of coronary heart disease (CHD) and cardiovascular diseases (CVDs) calculated by the Framingham Risk Score (FRS) models were reported for subjects with HIV and hepatitis $\mathrm{C}$ virus (HCV) infection. This novel study aimed to compare the CHD and CVDs risk factors as well CHD and CVDs events predictions using FRS between HIV- infected and HIV/HCV subjects on antiretroviral therapy (ART) in Malaysia.

Methods This retrospective study was conducted with a purposive sampling of 2046 HIV patients on ART in an outpatient clinic in Malaysia age, gender, lipid profile, blood pressure, smoking, diabetes status, immunity indices, and ART using digital medical records. Risks were predicted using FRS models (2002) for CHD and FRS formula (2008) for CVDs. Descriptive, independent sample T-test and Chi-square statistical tests were applied.

Results CHD and CVD risks were estimated in 1850 subjects (median age 46 years, 20\% female) only with HIV and 196 of HIV/HCV subjects (median age 43 years, $4 \%$ female). The HIV/ HCV group had significant lower mean levels of total cholesterol, HDL, LDL, triglyceride and systolic blood pressure while mean age was significant higher in HIV group $(\mathrm{p}<0.05)$. HCV/ HIV group had significant proportion of Tenofovir receivers $(34.6 \%$ vs $16.6 \%)$ and lower CD4 count level ( $p<0.05)$. The intermediate and high risks of CHD were prevalent among HIV/ HCV and HIV subjects as $6.1 \%$ vs $6.6 \%$ and $3.1 \%$ vs $3.6 \%$ respectively. Also HIV vs $\mathrm{HIV} / \mathrm{HCV}$ subjects had intermediate CVD risks as $40.9 \%$ vs $38.6 \%$ while $8.3 \%$ vs $8.2 \%$ had high risk CVDs. Points and risks percentages of CVDs and CHD were not significant different between $\mathrm{HIV}$ and $\mathrm{HIV} / \mathrm{HCV}$ groups.

Conclusion Risk of CHD and CVDs were similar in HIV and HIV/HCV groups. This Study suggests a need for more specific FRS equations since the serum lipid profiles influence by some factors such as immunity status, ART and HCV rather than traditional risks.

Disclosure of interest statement There is no conflict of interest.

\section{P16.28 FRAMINGHAM CORONARY HEART DISEASE AND CARDIOVASCULAR RISK ASSESSMENTS OF HIVIAIDS MALAYSIAN POPULATION ON HAART: THE IMPORTANCE OF RISKS EVALUATIONS AND PREDICTIONS}

${ }^{1} \mathrm{~N}$ Hejazi, ${ }^{1} \mathrm{R}$ Rajikan, ${ }^{2} \mathrm{CLK}$ Choong. 'National University of Malaysia; ${ }^{2}$ Sungai Buloh Hospital

\subsection{6/sextrans-2015-052270.575}

Background Coronary heart disease (CHD) and cardiovascular diseases (CVDs) events have increasing trends mainly due to the multiple and complex mechanisms of chronic inflammation and anti-retroviral drugs adverse effects during HIV course. Due to the lack of information this study aimed to analyse the CHD and CVDs risk profiles, estimate the probability of events and evaluate the accuracy of the Framingham CHD equations comprehensively in HIV-infected Malaysian subjects on highly active antiretroviral therapy (HAART).

Methods This is a cross-sectional study with a purposive sampling of 2046 HIV patients on HAART in an outpatient infectious disease clinic in Selangor Malaysia. Using digital medical records. all variables for Framingham equations including demographics, gender, fasting plasma glucose and lipid profiles, blood pressure, smoking and diabetes status, hypertension treatment, immunity indices and antiretroviral therapy were collected. 10years CHD risks were predicted using Framingham Risk Score (FRS1998 and FRS 2002) models while CVDs risk by specific FRS (2008). Data analyses included descriptive statistics and binary logistic regression. 